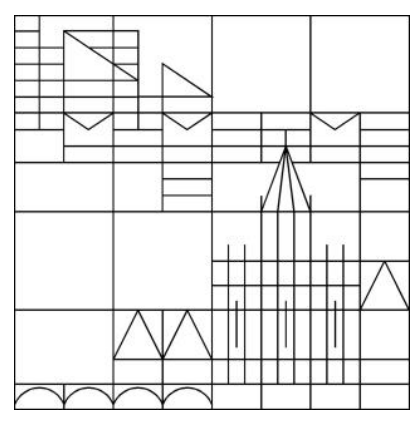

\title{
Reduced-Order Multiobjective Optimal Control of Semilinear Parabolic Problems
}

\author{
Laura Iapichino \\ Stefan Trenz \\ Stefan Volkwein
}

Konstanzer Schriften in Mathematik

Nr. 347, Dezember 2015

ISSN 1430-3558

Konstanzer Online-Publikations-System (KOPS)

URL: http://nbn-resolving.de/urn:nbn:de:bsz:352-0-313874

(C) Fachbereich Mathematik und Statistik

Universität Konstanz

Fach D 197, 78457 Konstanz, Germany 



\title{
Reduced-Order Multiobjective Optimal Control of Semilinear Parabolic Problems
}

\author{
Laura Iapichino ${ }^{1}$, Stefan Trenz ${ }^{2}$, and Stefan Volkwein ${ }^{3}$ \\ 1 Delft University of Technology, Department of Precision and Microsystems \\ Engineering, Mekelweg 2, 2628 CD Delft, The Netherlands, \\ 1.iapichino@tudelft.nl \\ 2 University of Konstanz, Department of Mathematics and Statistics, \\ Universitätsstraße 10, D-78457 Konstanz, Germany, \\ stefan.trenz@uni-konstanz.de \\ 3 University of Konstanz, Department of Mathematics and Statistics, \\ Universitätsstraße 10, D-78457 Konstanz, Germany, \\ stefan.volkwein@uni-konstanz.de
}

\begin{abstract}
In this paper a reduced-order strategy is applied to solve a multiobjective optimal control problem governed by semilinear parabolic partial differential equations. These problems often arise in practical applications, where the quality of the system behavior has to be measured by more than one criterium. The weighted sum method is exploited for defining scalar-valued nonlinear optimal control problems built by introducing additional optimization parameters. The optimal controls corresponding to specific choices of the optimization parameters are efficiently computed by the reduced-order method. The accuracy is guaranteed by an a-posteriori error estimate.
\end{abstract}

\section{Introduction}

In real applications, optimization problems are often described by introducing several objective functions conflicting with each other. This leads to multiobjective or multicriterial optimization problems; see, e.g., [1]. Finding the optimal control that represents a good compromise is the main issue in these problems. For that reason the concept of Pareto optimal or efficient points is developed. In contrast to scalar-valued optimization problems, the computation of a set of Pareto optimal points is required. Consequently, many scalar-valued constrained optimization problems have to be solved.

When dealing with control functions instead of parameters, a multiobjective optimal control problem (MOCP) needs to be solved. In this paper we apply the weighted sum method [14,1] in order to transform the MOCP into a sequence of scalar optimal control problems and to solve them using well known optimal control techniques [12]. Preliminary results combining reduced-order modeling and multiobjective PDE-constrained optimization are recently derived $[4,9]$. The main focus of the present work lies in the extension of [4] to nonlinear, control constrained optimal control problems governed by evolution problems. 
The paper is organized as follows. In Section 2 the multiobjective optimal control problem is formulated and transformed into a scalar-valued problem that is considered in Section 3. The numerical strategy and results are discussed in Section 4.

\section{Problem formulation and Pareto optimality}

Let $\Omega \subset \mathbb{R}^{\mathrm{d}}, \mathrm{d} \in\{1,2,3\}$, be an open and bounded domain with Lipschitz continuous boundary $\Gamma=\partial \Omega$. For given $T>0$ we set $Q=(0, T) \times \Omega$ and $\Sigma=(0, T) \times \Gamma$. Then we consider the following class of multiobjective optimal control problems governed by semilinear parabolic equations:

$$
\min \mathcal{J}(y, u)=\left(\begin{array}{c}
\mathcal{J}_{1}(y, u) \\
\mathcal{J}_{2}(y, u) \\
\mathcal{J}_{3}(y, u)
\end{array}\right)=\left(\begin{array}{c}
\frac{1}{2} \int_{\Omega}\left|y(T, \cdot)-y_{\Omega}\right|^{2} \mathrm{~d} \boldsymbol{x} \\
\frac{1}{2} \int_{0}^{T} \int_{\Omega}\left|y-y_{Q}\right|^{2} \mathrm{~d} \boldsymbol{x} \mathrm{d} t \\
\frac{1}{2} \sum_{i=1}^{m}\left|u_{i}-u_{i}^{d}\right|^{2}
\end{array}\right)
$$

subject to the semilinear parabolic differential problem

$$
\begin{aligned}
& y_{t}(t, \boldsymbol{x})-\Delta y(t, \boldsymbol{x})+y^{3}(t, \boldsymbol{x})=\sum_{i=1}^{m} u_{i} b_{i}(\boldsymbol{x})+f(t, \boldsymbol{x}) \text { for }(t, \boldsymbol{x}) \in Q, \\
& \frac{\partial y}{\partial n}(t, \boldsymbol{s})=0 \text { for }(t, \boldsymbol{s}) \in \Sigma, \quad y(0, \boldsymbol{x})=y_{\circ}(\boldsymbol{x}) \text { for } \boldsymbol{x} \in \Omega
\end{aligned}
$$

and to the bilateral control constraints

$$
u \in \mathcal{U}_{\mathrm{ad}}=\left\{\tilde{u}=\left(\tilde{u}_{1}, \ldots, \tilde{u}_{m}\right)^{\top} \in \mathbb{R}^{m} \mid u_{i}^{a} \leq \tilde{u}_{i} \leq u_{i}^{b} \text { for } 1 \leq i \leq m\right\} .
$$

In (1a) we assume that $y_{\Omega} \in L^{\infty}(\Omega), y_{Q} \in L^{\infty}(Q), u^{d}=\left(u_{1}^{d}, \ldots, u_{m}^{d}\right)^{\top} \in$ $\mathbb{R}^{m}$. By ' $T$ ' we denote the transpose of a vector or matrix. Furthermore, we suppose that $b_{1}, \ldots, b_{m} \in L^{\infty}(\Omega), y_{\circ} \in L^{\infty}(\Omega)$. In (1c) let $u_{a}^{i}, u_{b}^{i} \in \mathbb{R}$ satisfying $u_{a}^{i} \leq u_{b}^{i}$ for $1 \leq i \leq m$.

Recall that the state equation (1b) has a unique (weak) solution $y=y(u)$ for every $u \in \mathcal{U}_{\mathrm{ad}}$; see, e.g., [12]. Hence, we can introduce the reduced objective by $\hat{\mathcal{J}}(u)=\mathcal{J}(y(u), u)$ for $u \in \mathcal{U}_{\text {ad }}$. Instead of (1) we consider now

$$
\min \hat{\mathcal{J}}(u)=\left(\begin{array}{c}
\hat{\mathcal{J}}_{1}(u) \\
\hat{\mathcal{J}}_{2}(u) \\
\hat{\mathcal{J}}_{3}(u)
\end{array}\right) \quad \text { subject to } u \in \mathcal{U}_{\mathrm{ad}} .
$$

Problem (2) involves the minimization of a vector-valued objective. This is done by using the concepts of order relation and Pareto optimality [1]. In $\mathbb{R}^{3}$ we make use of the following order relation: For all $z^{1}, z^{2} \in \mathbb{R}^{3}$ we have

$$
z^{1} \leq z^{2} \quad \Leftrightarrow \quad z^{2}-z^{1} \in \mathbb{R}_{+}^{3}=\left\{z \in \mathbb{R}^{3} \mid z_{i} \geq 0 \text { for } i=1,2,3\right\} .
$$


Definition 1 (Pareto optimal). Let $z=\hat{\mathcal{J}}\left(\mathcal{U}_{\mathrm{ad}}\right) \subset \mathbb{R}^{3}$ be the image set of $\mathcal{U}_{\text {ad }}$ under the cost functional $\hat{\mathcal{J}}$. We call a point $\bar{z} \in \mathcal{Z}$ globally (strictly) efficient with respect to the order relation $\leq$, if there exists no $z \in Z \backslash\{\bar{z}\}$ with $z \leq \bar{z}$. If $\bar{z}$ is efficient and $\bar{u} \in \mathcal{U}_{\text {ad }}$ satisfies $\bar{z}=\hat{\mathcal{J}}(\bar{u})$, we call $\bar{u}$ (strictly) Pareto optimal. Let $\bar{u} \in \mathcal{U}_{\mathrm{ad}}$ hold. If there exists a neighborhood $\mathcal{N}(\bar{u}) \subset \mathfrak{U}_{\mathrm{ad}}$ of $\bar{u}$ so that $\bar{z}=\hat{\mathcal{J}}(\bar{u})$ is (strictly) efficient for the (local) image set $\hat{\mathcal{J}}(\mathcal{N}(\bar{u})) \subset \mathcal{Z}$, the point $\bar{u}$ is called locally (strictly) Pareto optimal. Moreover, $\bar{z}$ is said to be locally efficient.

Now, the multiobjective optimal control problem (2) is understood as follows: Find Pareto optimal points in $\mathfrak{U}_{\text {ad }}$ for the vector-valued reduced objective $\hat{\mathcal{J}}$.

First-order necessary optimality conditions for Pareto optimality are presented in the next theorem which is proved in [1, Theorem 3.21 and Corollary 3.23]. The proof is based on the result of Kuhn-Tucker [6].

Theorem 2. Suppose that $\bar{u} \in \mathcal{U}_{\mathrm{ad}}$ is Pareto optimal. Then, there exists a parameter vector $\boldsymbol{\mu}=\left(\bar{\mu}_{1}, \bar{\mu}_{2}, \bar{\mu}_{3}\right) \in \mathbb{R}^{3}$ satisfying the Karush-Kuhn-Tucker conditions

$$
\bar{\mu}_{i} \in[0,1], \quad \sum_{i=1}^{3} \bar{\mu}_{i}=1 \text { and } \sum_{i=1}^{3} \bar{\mu}_{i} \hat{\mathcal{J}}_{i}^{\prime}(\bar{u})^{\top}(u-\bar{u}) \geq 0 \text { for all } u \in \mathcal{U}_{\text {ad }} .
$$

Motivated by Theorem 2 , let us choose $0 \ll \mu_{l b}<1$ and set

$$
\mathcal{D}_{\mathrm{ad}}=\left\{\boldsymbol{\mu}=\left(\mu_{1}, \mu_{2}, \mu_{3}\right) \in \mathbb{R}_{+}^{k} \mid \sum_{i=1}^{3} \mu_{i}=1, \mu_{3} \geq \mu_{l b}\right\} \subset[0,1]^{3} .
$$

The condition $\mu_{3} \geq \mu_{l b}$ is necessary for the well-posedness of the scalarvalued optimal problem $\left(\hat{\mathbf{P}}_{\boldsymbol{\mu}}\right)$ introduced below. For any $\boldsymbol{\mu} \in \mathcal{D}_{\text {ad }}$ we define the parameter-dependent, scalar-valued objective as $\hat{J}(u ; \boldsymbol{\mu})=\boldsymbol{\mu}^{\top} \hat{\mathcal{J}}(u)$ for $u \in \mathcal{U}_{\text {ad }}$. Then, (3) are the first-order necessary optimality conditions for a local solution $\bar{u}=\bar{u}(\boldsymbol{\mu})$ to the parameter-dependent optimization problem

$$
\min \hat{J}(u ; \boldsymbol{\mu}) \quad \text { subject to } \quad u \in \mathcal{U}_{\text {ad }} \quad\left(\hat{\mathbf{P}}_{\boldsymbol{\mu}}\right)
$$

for the parameter $\boldsymbol{\mu}=\overline{\boldsymbol{\mu}}$. In the weighted sum method Pareto optimal points are computed by solving $\left(\hat{\mathbf{P}}_{\boldsymbol{\mu}}\right)$ for various $\boldsymbol{\mu} \in \mathcal{D}_{\text {ad }}$; see [14] and [1, Chapter 3].

Remark 3. To solve $\left(\hat{\mathbf{P}}_{\boldsymbol{\mu}}\right)$ we apply a globalized Newton-CG method [7]. $\diamond$

\section{The scalar-valued optimal control problem}

Suppose that $\bar{u}=u(\boldsymbol{\mu}) \in \mathcal{U}_{\mathrm{ad}}$ is an optimal solution to $\left(\hat{\mathbf{P}}_{\boldsymbol{\mu}}\right)$ for given $\boldsymbol{\mu} \in$ $\mathcal{D}_{\text {ad }}$. Let $\bar{y}=y(\bar{u} ; \boldsymbol{\mu})$ denote the associated optimal state satisfying (1b) for 
$u=\bar{u}$. Following [12] first-order necessary optimality conditions for $\left(\hat{\mathbf{P}}_{\boldsymbol{\mu}}\right)$ ensure the existence of a unique adjoint $\bar{p}=p(\bar{u} ; \boldsymbol{\mu})$ solving

$$
\begin{aligned}
& -p_{t}(t, \boldsymbol{x})-\Delta p(t, \boldsymbol{x})+3 y^{2}(t, \boldsymbol{x}) p(t, \boldsymbol{x})=\mu_{1}\left(y_{Q}(t, \boldsymbol{x})-\bar{y}(t, \boldsymbol{x})\right) \text { in } Q \\
& \frac{\partial p}{\partial n}(t, \boldsymbol{s})=0 \text { on } \Sigma, \quad p(T, \boldsymbol{x})=\mu_{2}\left(y_{\Omega}(\boldsymbol{x})-\bar{y}(T, \boldsymbol{x})\right) \text { in } \Omega
\end{aligned}
$$

with $y=\bar{y}$. Moreover, for any $\boldsymbol{\mu} \in \mathcal{D}_{\text {ad }}$ the gradient of the reduced cost functional $\hat{J}(\cdot ; \boldsymbol{\mu})$ at a given $u \in \mathcal{U}_{\text {ad }}$ is given by

$$
\hat{J}^{\prime}(u ; \boldsymbol{\mu})=\left(\mu_{3}\left(u_{i}-u_{i}^{d}\right)-\int_{0}^{T} \int_{\Omega} p(t, \boldsymbol{x}) b_{i}(\boldsymbol{x}) \mathrm{d} \boldsymbol{x} \mathrm{d} t\right)_{1 \leq i \leq m},
$$

where $p$ solves (4) and $y$ is the solution to (1b).

Since $\left(\hat{\mathbf{P}}_{\boldsymbol{\mu}}\right)$ is a non-convex problem, we make use of the hessian $\hat{J}^{\prime \prime}(u ; \boldsymbol{\mu}) \in$ $\mathbb{R}^{m \times m}$ in order to ensure sufficient optimality conditions. Let $\bar{u}=\bar{u}(\boldsymbol{\mu})$ be locally optimal for $\left(\hat{\mathbf{P}}_{\boldsymbol{\mu}}\right)$ satisfying the second-order sufficient optimality condition

$$
\hat{J}^{\prime \prime}(\bar{u} ; \boldsymbol{\mu})(u, u) \geq \kappa|u|_{2}^{2} \quad \text { for all } u \in \mathbb{R}^{m}
$$

with a $\boldsymbol{\mu}$-independent lower bound $\kappa>0$ for the smallest eigenvalue of the hessian. Then, for any $\tilde{\kappa} \in(0, \kappa)$ there exists a radius $\tilde{\rho}=\rho(\tilde{\kappa})>0$ such that

$$
\hat{J}^{\prime \prime}(\tilde{u} ; \boldsymbol{\mu})(u, u) \geq \tilde{\kappa}|u|_{2}^{2} \text { for all } \tilde{u} \text { with }|\tilde{u}-\bar{u}|_{2} \leq \tilde{\rho} .
$$

If $\tilde{u} \in \mathcal{U}_{\text {ad }}$ satisfies (5) we can estimate the error between $\bar{u}$ and $\tilde{u}$ as [5]

$$
|\bar{u}-\tilde{u}|_{2} \leq \frac{1}{\tilde{\kappa}}|\zeta|_{2}
$$

where $\zeta=\zeta(\tilde{u}) \in \mathbb{R}^{m}$ is given by

$$
\zeta_{i}:=\left\{\begin{array}{cl}
{\left[\mu_{3}\left(u_{i}-u_{i}^{d}\right)-\int_{0}^{T} \int_{\Omega} \tilde{p}(t, \boldsymbol{x}) b_{i}(\boldsymbol{x}) \mathrm{d} \boldsymbol{x} \mathrm{d} t\right]_{-},} & \text {if } \tilde{u}_{i}=u_{i}^{a}, \\
-\mu_{3}\left(u_{i}-u_{i}^{d}\right)+\int_{0}^{T} \int_{\Omega} \tilde{p}(t, \boldsymbol{x}) b_{i}(\boldsymbol{x}) \mathrm{d} \boldsymbol{x} \mathrm{d} t, & \text { if } u_{i}^{a}<\tilde{u}_{i}<u_{i}^{b}, \\
-\left[\mu_{3}\left(u_{i}-u_{i}^{d}\right)-\int_{0}^{T} \int_{\Omega} \tilde{p}(t, \boldsymbol{x}) b_{i}(\boldsymbol{x}) \mathrm{d} \boldsymbol{x} \mathrm{d} t\right]_{+}, & \text {if } \tilde{u}_{i}=u_{i}^{b},
\end{array}\right.
$$

$\tilde{p}$ solves (4) with $y=\tilde{y}$ and $\tilde{y}$ solves (1b) with $u=\tilde{u}$. In (7) we denote by $[s]_{-}=-\min (0, s)$ and $[s]_{+}=\max (0, s)$ the negative and positive part function, respectively. Hence, given a (suboptimal) $\tilde{u} \in \mathcal{U}_{\mathrm{ad}}$, the error $\bar{u}-\tilde{u}$ can be estimated by the right-hand side in (5) provided the lower bound $\tilde{\kappa}$ for the symmetric matrix $\hat{J}^{\prime \prime}(\tilde{u} ; \boldsymbol{\mu})$ is known. We proceed as follows: From $\hat{J}(\tilde{u} ; \boldsymbol{\mu})=\boldsymbol{\mu}^{\top} \hat{\mathcal{J}}(\tilde{u})$ we find

$$
\hat{J}^{\prime \prime}(\tilde{u} ; \boldsymbol{\mu})=\mu_{1} \hat{\mathcal{J}}_{1}^{\prime \prime}(\tilde{u})+\mu_{2} \hat{\mathcal{J}}_{2}^{\prime \prime}(\tilde{u})+\mu_{3} \hat{\mathcal{J}}_{3}^{\prime \prime}(\tilde{u}) \quad \text { for } \boldsymbol{\mu} \in \mathcal{D}_{\mathrm{ad}} .
$$


It follows from Theorem of Courant-Fischer [10, Corollary 4.7] that a lower bound $\lambda_{\min }^{\mathrm{LB}}$ for the smallest eigenvalue $\lambda_{\min }$ of $\hat{J}^{\prime \prime}(\tilde{u} ; \boldsymbol{\mu})$ is given by

$$
\lambda_{\min }\left(\hat{J}^{\prime \prime}(\tilde{u} ; \boldsymbol{\mu})\right) \geq \sum_{i=1}^{3} \mu_{i} \lambda_{\min }\left(\hat{\mathcal{J}}_{i}^{\prime \prime}(\tilde{u})\right)=: \quad \lambda_{\min }^{\mathrm{LB}}(\tilde{u} ; \boldsymbol{\mu}),
$$

where $\lambda_{\min }(A)$ denotes the smallest eigenvalue of a symmetric matrix $A$.

\section{Numerical solution strategy}

To solve (1) we apply the weighted sum method. Thus, the set of Pareto optimal points is approximated by solutions $\bar{u}(\boldsymbol{\mu})$ to $\left(\hat{\mathbf{P}}_{\boldsymbol{\mu}}\right)$ for various parameters $\boldsymbol{\mu} \in \mathcal{D}_{\text {ad }}$. Consequently, many constrained nonlinear optimization problems have to be solved numerically, which is computationally expensive. For this reason model-order reduction (MOR) is applied to reduce significantly the required computational resources. Our MOR approach is based on a Galerkintype approximation to $\left(\hat{\mathbf{P}}_{\boldsymbol{\mu}}\right)$ using MOR basis functions, where for certain weighting parameters $\boldsymbol{\mu} \in \mathcal{D}_{\text {ad }}$ the MOR basis functions contain information from optimal states $\bar{y}(\boldsymbol{\mu})$ and adjoints $\bar{p}(\boldsymbol{\mu})$ associated with optimal controls $\bar{u}(\boldsymbol{\mu})$. The MOR basis functions are determined in an offline phase. In the online phase the weighted sum method is applied, where numerical solutions to $\left(\hat{\mathbf{P}}_{\boldsymbol{\mu}}\right)$ are computed rapidly by a MOR Galerkin discretization [11].

Offline phase I: eigenvalue computation on control grid. Suppose the discrete (regular) control grid $\Xi_{\text {grid }}=\left\{u^{k}\right\}_{k=1}^{K}$ in the set $\mathcal{U}_{\text {ad }}$ of admissible controls. In an offline phase we compute and store the $\boldsymbol{\mu}$-independent smallest eigenvalues $\lambda_{\min }\left(\hat{\mathcal{J}}_{i}^{\prime \prime}\left(u^{k}\right)\right)$ at any grid node $u^{k} \in \Xi_{\text {grid }}$ for $i=1$ and 2. Since $\hat{\mathcal{J}}_{3}^{\prime \prime}\left(u^{k}\right)$ is the identity, we have $\lambda_{\min }\left(\hat{\mathcal{J}}_{3}^{\prime \prime}\left(u^{k}\right)\right)=1$. Now, (8) yields a numerically efficient computation of the approximative lower bound $\lambda_{\text {app }}^{\mathrm{LB}}(\tilde{u} ; \boldsymbol{\mu})$ in the online phase at any (suboptimal) control $\tilde{u} \in \mathcal{U}_{\text {ad }}$ by convex combination of the stored smallest eigenvalues $\lambda_{\min }\left(\hat{\mathcal{J}}_{i}^{\prime \prime}\left(u^{k}\right)\right)$ for $k=1, \ldots, K$ :

$$
\lambda_{\min }\left(\hat{\mathcal{J}}_{i}^{\prime \prime}(\tilde{u})\right) \approx \lambda_{\text {app }}^{i}(\tilde{u}):=\sum_{k=1}^{K} \omega_{k} \lambda_{\min }\left(\hat{\mathcal{J}}_{i}^{\prime \prime}\left(u^{k}\right)\right) .
$$

In (6) we utilize $\lambda_{\text {app }}^{i}(\tilde{u})$ instead of $\tilde{\kappa}$.

Remark 4. The computation of the $\lambda_{\min }\left(\hat{\mathcal{J}}_{i}^{\prime \prime}\left(u^{k}\right)\right)$ can be realized in parallel computing with respect to $k \in\{1, \ldots, K\}$.

Offline phase II: MOR basis computation. Estimate (6) can be suitably used as ingredient to apply a MOR strategy for the solution of nonlinear multiobjective problems. In order to use a MOR technique for its solution, we 
propose to use the POD-greedy algorithm based on [3] and [2,8]. As an input the POD-greedy algorithm requires a discrete parameter training set $S_{\text {train }} \subset$ $\mathcal{D}_{\text {ad }}$, as well as he smallest eigenvalues $\lambda_{\text {min }}\left(\hat{\mathcal{J}}_{i}^{\prime \prime}\left(u^{k}\right)\right)$ for $i=1,2,3$ on the control grid $\Xi_{\text {grid }}$ and the corresponding precomputed grid node data $D_{\text {grid }}$, both needed for the smallest eigenvalue approximation in the a-posteriori error estimation.

Online phase: multiobjective optimal control As regards the original multiobjective problem, we are interested in the solution of the parametric optimal control problem for a large number of parameter values, since we want to identify a set of optimal control solutions that does not a-priorily penalize any cost functional. In other words, we are interested in identifying the Pareto optimal front of the multiobjective problem, that consists in a large set of cost functionals evaluation corresponding to the solution of a large number of optimal control problems (obtained in correspondence of several parameter values, at the randomly chosen). In order to identify the Pareto front we need to evaluate several times the parametric optimal control problem. For this purpose, the proposed model order reduction strategy can be efficiently reduce the required computational times.

\section{Numerical example}

We consider (1) with spatial domain $\Omega=(0,1) \times(0,1) \subset \mathbb{R}^{2}$, final time $T=1$, desired states $y_{\Omega}=0, y_{Q}(t, \boldsymbol{x})=100 t \cos \left(2 \pi x_{1}\right) \cos \left(2 \pi x_{2}\right)$, initial condition $y_{\circ}(\boldsymbol{x})=0$ and inhomogeneity $f(t, \boldsymbol{x})=10 t x_{1}$. Furthermore, for $m=4$ each shape function $b_{i}(\boldsymbol{x}), i=1, \ldots, 4$, has the support in a quarter of the domain $\Omega$ and $u^{d}=(0.5,-4,-0.5,4)^{\top} \in \mathbb{R}^{4}$. The high fidelity spatial approximations of the problem solution, used for the basis computations and the error comparisons is computed by a finite element (FE) model with a Newton method that uses $\mathbb{P}_{1}$ elements, it has 729 degrees of freedom in 1352 elements. For the temporal discretization the implicit Euler method is applied with equidistant step size $\Delta t=0.01$ steps. Figure 1 shows optimal states $\bar{y}=y(\bar{u} ; \boldsymbol{\mu})$ for solutions $\bar{u}(\boldsymbol{\mu})$ to $\left(\hat{\mathbf{P}}_{\boldsymbol{\mu}}\right)$ corresponding to two values of the parameter $\boldsymbol{\mu}$.In the left plot of Figure 2 we plot error comparisons obtained by solving $\left(\hat{\mathbf{P}}_{\boldsymbol{\mu}}\right)$. In particular, we compare the errors between the optimal controls computed by the model order reduction (MOR) method and the one by the FE method. We show its minimum, maximum and average values (over a range of 1000 parameter values) by varying the number of basis functions used in the MOR scheme. In the right plot of Figure 2 we present the Pareto front obtained by solving $\left(\hat{\mathbf{P}}_{\boldsymbol{\mu}}\right)$ with the MOR technique for different parameter values $\boldsymbol{\mu}$. In order to show the correctness of the Pareto front, we also include the values of the cost functionals $\hat{\mathcal{J}}_{1}(u)$, $\hat{\mathcal{J}}_{2}(u)$ and $\hat{\mathcal{J}}_{3}(u)$ obtained for 1000 control values randomly chosen as follows: $u_{1} \in[-3,3], u_{2} \in[-8,-1], u_{3} \in[-5,-2], u_{4} \in[-1,6]$ (not optimal 

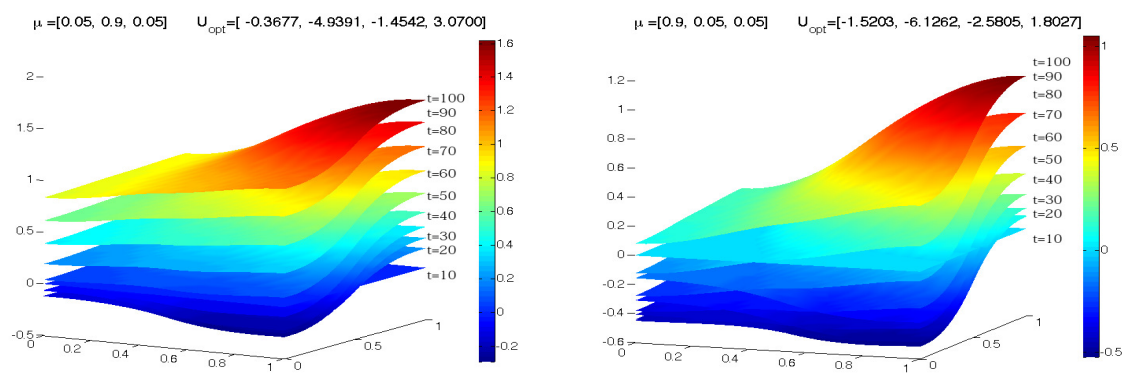

Fig. 1. Optimal states $\bar{y}=y(\bar{u} ; \boldsymbol{\mu})$ for solutions $\bar{u}(\boldsymbol{\mu})$ to $\left(\hat{\mathbf{P}}_{\boldsymbol{\mu}}\right)$ for parameter values $\boldsymbol{\mu}=(0.05,0.9,0.05)$ (left) and $\boldsymbol{\mu}=(0.9,0.05,0.05)$ (right).
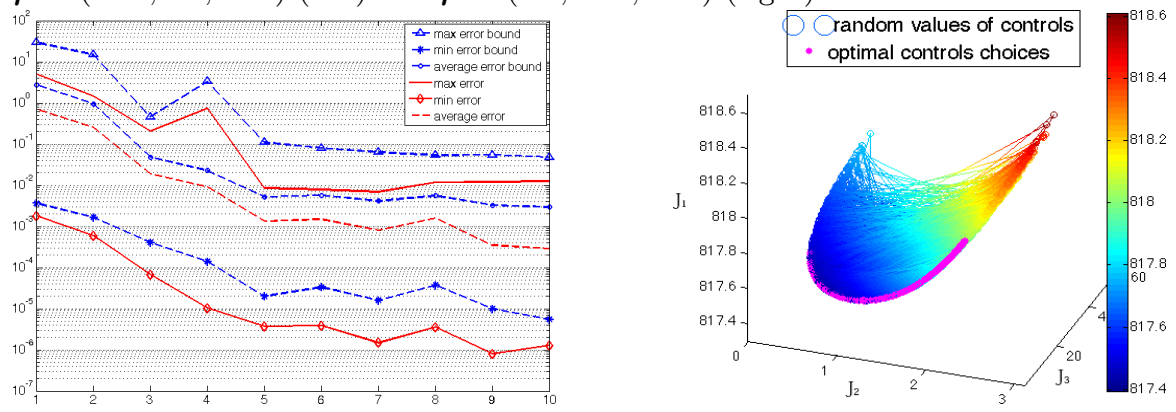

Fig. 2. Error bound and error between the MOR and FE solutions by varying the number of bases: minimum, maximum and average values over a set of 100 random parameter values (left); Pareto front and cost functionals values corresponding to admissible control points (right).

controls). In Figure 3 we show the optimal controls $\bar{u}(\boldsymbol{\mu})$ corresponding to 1000 randomly chosen parameter values and to the parameter values selected for the bases computations during the POD-greedy algorithm. Regarding the computational performances the online evaluation time required for solving problem $\left(\hat{\mathbf{P}}_{\boldsymbol{\mu}}\right)$ for each parameter value by using 10 basis functions is about 0.7 seconds; while the evaluation of the $\mathrm{FE}$ solution requires about $7.3 \mathrm{sec}-$ onds. We conclude that the gained speedup allows a much faster optimal solution evaluation and an efficient identification of the Pareto front, for which several repeated solutions have to be computed.

\section{References}

1. M. Ehrgott. Multicriteria Optimization Springer, Berlin, 2005.

2. M. Grepl. Certified reduced basis methods for nonaffine linear time-varying and nonlinear parabolic partial differential equations. Mathematical Models and Methods in Applied Sciences; 22(3), 2012. 

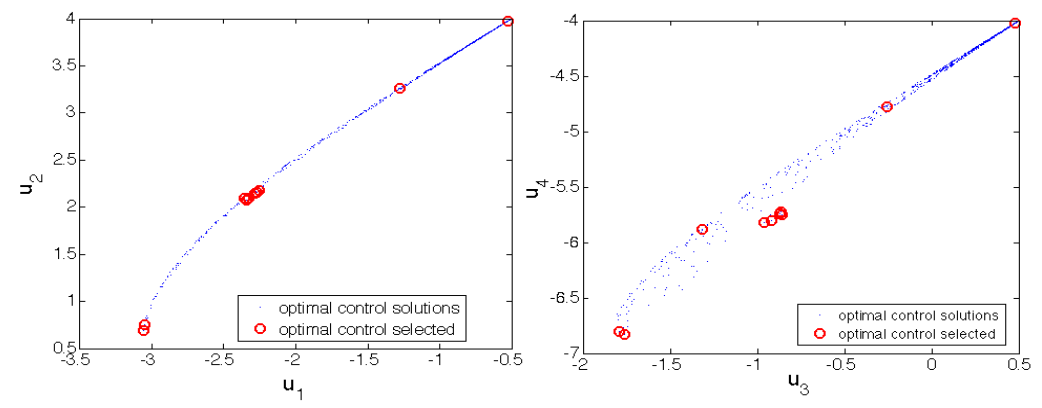

Fig. 3. Optimal controls $\bar{u}(\boldsymbol{\mu})$ corresponding to 1000 randomly chosen parameter values (left) and to the parameter values selected for the bases computations during the POD-greedy algorithm (right).

3. B. Haasdonk and M. Ohlberger. Reduced basis method for finite volume approximations of parametrized linear evolution equations. Mathematical Modelling and Numerical Analysis; 42; pp. 277-302, 2008.

4. L. Iapichino, S. Ulbrich, and S. Volkwein. Multiobjective PDE-constrained optimization using the reduced-basis method. Submitted (2015), available at http://nbn-resolving.de/urn:nbn:de:bsz:352-2501909

5. E. Kammann, F. Tröltzsch, S. Volkwein. A-posteriori error estimation for semilinear parabolic optimal control problems with application to model reduction by POD. Mathematical Modelling and Numerical Analysis; 47; pp. 555-581, 2013.

6. H. Kuhn and A. Tucker. Nonlinear programming. In Newman, J., editor, Proceedings of the Second Berkeley Symposium on Mathematical Statistics and Probability, University of California Press, Berkeley, pp. 481-492, 1951.

7. J. Nocedal and S.J. Wright. Numerical Optimization. Springer Series in Operation Research, second edition, 2006.

8. A.T. Patera and G. Rozza. Reduced Basis Approximation and A Posteriori Error Estimation for Parametrized Partial Differential Equations. MIT, 2007.

9. S. Peitz and M. Dellnitz. Multiobjective optimization of the flow around a cylinder using model order reduction. In Proceedings in Applied Mathematics and Mechanics (PAMM) - Accepted, 2015.

10. G.W. Stuart and J. Sun. Matrix Perturbation Theory. Computer Science and Scientific Computing. Academic Press, 1990.

11. E. Sachs and S. Volkwein. POD Galerkin approximations in PDE-constrained optimization. GAMM-Mitteilungen; 33; pp. 194-208, 2010

12. Tröltzsch, F.: Optimal Control of Partial Differential Equations: Theory, Methods and applications. Graduate Studies in Mathematics, Vol. 112, American Mathematical Society (2010)

13. F. Tröltzsch and S. Volkwein. POD a-posteriori error estimates for linearquadratic optimal control problems. Computational Optimization and Applications; 44; pp. 83-115, 2009.

14. L. Zadeh. Optimality and non-scalar-valued performance criteria. IEEE Transactions on Automatic Control; 8; pp. 59-60, 1963. 